\title{
Ankle Joint Dorsiflexion Measurement Using the Deep Squatting Posture
}

\author{
TATSUYA KasUyAma, RPT, MS ${ }^{1,2)}$, MASAAKI SAKAMOTO, RPT, $\mathrm{PhD}^{2)}$, \\ RIE NAKAZAWA, RPT, $\mathrm{PhD}^{3}$ ) \\ 1)Asakura Clinic, Seseragi Hospital: 249-1 Asakura-machi, Maebashi, Gunma 371-0081, \\ Japan. TEL: +81 27-265-6522, FAX: +81 27-265-6527, \\ E-mail: kasuyama@health.gunma-u.ac.jp \\ ${ }^{2)}$ Graduate School of Medicine, Gunma University \\ ${ }^{3)}$ School of Medicine, Gunma University
}

J. Phys. Ther. Sci.

21: 195-199, 2009

\begin{abstract}
Purpose] The purpose of this study was to clarify the intrinsic factors influencing the deep squatting posture and to investigate the utility of ankle joint dorsiflexion measurement using this posture. [Subjects] The study subjects were 71 healthy male individuals. [Methods] The subjects were asked to squatted with their heels down and then were divided into 2 groups: possible squatting and impossible squatting. The anthropometric characteristics of the subjects were assessed, and the flexibility and movement range of the lower extremities were tested. To identify the intrinsic factors influencing the deep squatting posture, a dediscriminant analysis was performed by a stepwise procedure. The sensitivity, specificity, and cutoff values for the factors were evaluated by receiver operating characteristic curve analysis. [Results] Of the 71 participants, 55 were assigned to the possible squatting group and the remaining to the impossible squatting group. Analysis revealed that body weight and ankle dorsiflexion flexibility were significantly associated with ability to assume the deep squatting posture. In particular, ankle dorsiflexion flexibility was strongly associated with the ability to assume this posture. [Conclusion] The impossible squatting group showed reduced ankle dorsiflexion. Thus, the deep squatting posture is useful for easy and objective method for measuring ankle joint dorsiflexion flexibility.
\end{abstract}

Key words: Ankle joint dorsiflexion, Deep squatting posture, Physical therapy evaluation

(This article was submitted Dec. 18, 2008, and was accepted Feb. 17, 2009)

\section{INTRODUCTION}

Adequate flexibility in ankle dorsiflexion is necessary for normal performance of functional activities such as maintenance of gait, stair climbing, and rising from a chair ${ }^{1,2)}$. Limitation of ankle dorsiflexion is associated with gait parameters $^{3)}$ and balance function ${ }^{4)}$. In addition, it is considered to be a risk factor of falls for older people ${ }^{5)}$ and sports injuries in healthy males ${ }^{6-8)}$. The measurement method described herein is expected to enable the prediction of the risk factor, thus leading to the prevention of the risk of falls, sports injuries, etc. An easy and objective method for measuring ankle flexibility is necessary in physical therapy practice.

The deep squatting posture is adopted by workers on farms in welding units, automobile assembly plants, etc ${ }^{9}$. The deep squatting posture can be described as a sitting posture with dorsiflexed ankles, deeply flexed knees and hips, and a flexed torso, with the shoulder occasionally resting on the knee. A previous study reported that approximately $20 \%$ of Japanese failed to assume the deep squat posture perfectly on a Japanese-style toilet, and noted that the movement of the left and right ankle 
joints differed during squatting ${ }^{10)}$. The variations in squatting posture indicate high degrees of flexion of all the 3 joints of the lower extremities ${ }^{11)}$. In particular, ankle dorsiflexion flexibility is significantly correlated with the maintenance of a stable deep squatting posture ${ }^{10}$. Furthemore, body mass index (BMI) was found to be correlated to the maximal knee flexion during squatting ${ }^{12}$.

In this study, we were able to evaluate ankle dorsiflexion flexibility by using a simple method based on the deep squatting posture. However, it was necessary to clarify the influence of anthropometric characteristics and the flexibility of other joints on the ability to adopt the deep squatting posture.

The purpose of this study was to clarify the intrinsic factors influencing the deep squatting posture by descriminant analysis and to investigate the utility of ankle joint dorsiflexion measurement using this posture in healthy males.

\section{SUBJECTS AND METHODS}

\section{Subjects}

The study subjects were 71 healthy male college students who signed the consent forms after the study procedure was explained to them in detail. Their mean age was $21.0 \pm 2.9$ years; mean height, $170.8 \pm 5.8 \mathrm{~cm}$; mean body weight, $64.8 \pm 6.9 \mathrm{~kg}$; and mean BMI, $22.2 \pm 2.4 \mathrm{~kg} / \mathrm{m}^{2}$. None of the subjects had had musculoskeletal disorders in the lower extremity in the last 6 months.

\section{Methods}

The subjects were asked to squat with their heels down and then divided into 2 groups: possible squatting and impossible squatting. The former group comprised individuals who were able to squat, and the latter group comprised those who were unable to squat. The subjects were asked to sit in the deep squatting posture with their heels down and arms crossed and maintain the posture for more than 5 seconds. Two points to be kept in mind while squatting were that (1) both the knees and feet should be brought together to the maximum possible extent throughout the deep squat and (2) the thigh and calf should be in contact with each other.

The anthropometric characteristics of the subjects were assessed, and the flexibility and movement range of the lower extremities were tested. The anthropometric characteristics included body height, body weight, and BMI. It was necessary to measure the flexibility of each joint of the lower extremities in the deep squatting posture. Therefore, items reflecting the flexibility of each joint were measured by specific tests. The straight leg raise (SLR) test was used to measure hip flexibility ${ }^{13}$. In the SLR test, the subject's leg-with the knee held straight-was raised parallel to the edge of the table with the subject in the supine position, and the hip flexion angle was measured. Heel-buttock distance (HBD) was measured as an indicator of knee flexibility ${ }^{13)}$. HBD is the distance between the heel and the buttocks. The subject was placed in the prone position, and HBD was measured using a tape measure with the subject's knee passively bent. The modified Thomas test was used to measure the flexibility of the hip and pelvis ${ }^{14)}$. In the modified Thomas test, the subject sat at the end of a plinth and held both knees to the chest. The subject held one leg in maximal hip flexion with his arms, while the tested limb was lowered toward the floor. Flexibility was determined by measuring the hip flexion angle. Finger-floor distance (FFD) was measured as an indicator of trunk flexibility ${ }^{15)}$. FFD is the distance between the fingertip and the floor when the subject bends in an upright standing position and extends his fingers toward the floor. Ankle dorsiflexion flexibility was measured using the weight-bearing lunge test ${ }^{16)}$. A weight-bearing lunge is the distance between the tip of the big toe and the wall when the subject lunges toward the wall. It was measured with a tape measure placed on the floor.

Statistical analyses were performed with SPSS version 11.0J for windows. To examine the possible differences between the 2 squatting groups with regard to each test parameter, we used either Student's $t$ test (if the distribution of the data was normal) or the Mann-Whitney $U$ test (if the distribution of the data was not normal). A $p$ value of less than 0.05 was considered significant. Dependent variables for the deep squatting posture were analyzed by a stepwise linear discriminant analysis to determine their relative importance for differentiating between the 2 groups.

To show the significant association of the measured items with the intrinsic factors influencing the deep squatting posture, a receiver operator characteristic (ROC) curve was calculated. This curve was used to investigate the screening 
Table 1. Anthropometric characteristics of the subjects and the flexibilities and movement range of the lower extremities in both groups

\begin{tabular}{|c|c|c|c|}
\hline & $\begin{array}{l}\text { Possible Group } \\
\quad(\text { in }=55)\end{array}$ & $\begin{array}{l}\text { Impossible Group } \\
(\mathrm{n}=16)\end{array}$ & $\mathrm{p}$ value \\
\hline Age (years) & $20.9 \pm 2.0$ & $21.3 \pm 1.8$ & 0.556 \\
\hline Body Height (cm) & $170.1 \pm 5.5$ & $172.8 \pm 6.8$ & 0.064 \\
\hline Body Weight (kg) & $63.7 \pm 6.5$ & $68.6 \pm 7.0$ & $0.012 *$ \\
\hline Body Mass Index $\left(\mathrm{kg} / \mathrm{m}^{2}\right)$ & $22.0 \pm 2.4$ & $22.9 \pm 2.2$ & 0.211 \\
\hline Right Straight Leg Raise $\left(^{\circ}\right)$ & $68.7 \pm 7.4$ & $65.0 \pm 8.9$ & 0.096 \\
\hline Left Straight Leg Raise $\left(^{\circ}\right)$ & $67.5 \pm 7.6$ & $64.4 \pm 10.1$ & 0.120 \\
\hline Right Heel-Buttock Distance (cm) & $5.0 \pm 2.2$ & $6.7 \pm 3.2$ & $0.019 *$ \\
\hline Left Heel-Buttock Distance $(\mathrm{cm})$ & $5.6 \pm 2.7$ & $7.0 \pm 3.4$ & 0.080 \\
\hline Modified Thomas Test Values for the Right $\operatorname{Side}\left({ }^{\circ}\right)$ & $9.2 \pm 4.5$ & $10.0 \pm 4.1$ & 0.521 \\
\hline Modified Thomas Test Values for the Left Side $\left({ }^{\circ}\right)$ & $9.5 \pm 4.7$ & $10.0 \pm 4.2$ & 0.729 \\
\hline Finger-Floor Distance $(\mathrm{cm})$ & $3.2 \pm 10.6$ & $0.9 \pm 11.4$ & 0.455 \\
\hline Weight-bearing Lunge for the Right side $(\mathrm{cm})$ & $12.7 \pm 2.3$ & $8.6 \pm 2.8$ & $\mathrm{p}<0.001 * *$ \\
\hline Weight-bearing Lunge for the Left side $(\mathrm{cm})$ & $12.9 \pm 2.3$ & $8.1 \pm 3.1$ & $\mathrm{p}<0.001 * *$ \\
\hline
\end{tabular}

Mean \pm standard deviation (SD) .

*Significant difference between possible squatting and impossible squatting groups $(\mathrm{p}<0.05)$.

**Significant difference between possible squatting and impossible squatting groups $(\mathrm{p}<0.01)$.

Table 2. Results of the discriminant analysis

\begin{tabular}{lc}
\hline & Model $^{\text {a) }}$ \\
\hline Predictor Variable $^{\mathrm{b})}$ & Left Ankle Dorsiflexion Flexibility $(0.94)$ \\
& Body Weight $(-0.54)$ \\
Wilk's Lambda & $0.57^{*}$ \\
Canonical Correlation & 0.65 \\
Classification accuracyc) & $83 \%(81 \%)$ \\
\hline
\end{tabular}

a)Discriminant model considering the anthropometric characteristics and flexibility variables.

b) Significant predictor variables, with standard discriminant coefficients in brackets.

$* \mathrm{p}<0.05$.

c) Percentage of cases correctly classified, with cross-validated classification accurary shown in parenthesis.

point at which the groups showed the most difference with regard to the intrinsic factors influencing the deep squatting posture.

\section{RESULTS}

Of the 71 participants, 55 were determined belong to the possible squatting group, and the remaining 16 , to the impossible squatting group. Table 1 compares the anthropometric characteristics of the subjects and the flexibilities and movement range of the lower extremities in both the groups. Significant differences were observed in body weight, right HBD, and both right and left weight-bearing lunges $(\mathrm{p}<0.05)$. Table 2 shows the results of the discriminant analysis. The analysis revealed that left ankle dorsiflexion flexibility and body weight were significantly associated with ability to adopt the deep squatting posture. Ankle dorsiflexion flexibility, in particular, was strongly associated with the posture. ROC analysis was performed to investigate the association between ankle dorsiflexion flexibility and the ability to adopt the deep squatting posture. Figure 1 shows the ROC curves for the possible cutoff scores of the right and left ankle dorsiflexion flexibilities. The area under the ROC curves was 0.86 for the right side and 0.85 for the left side. The 

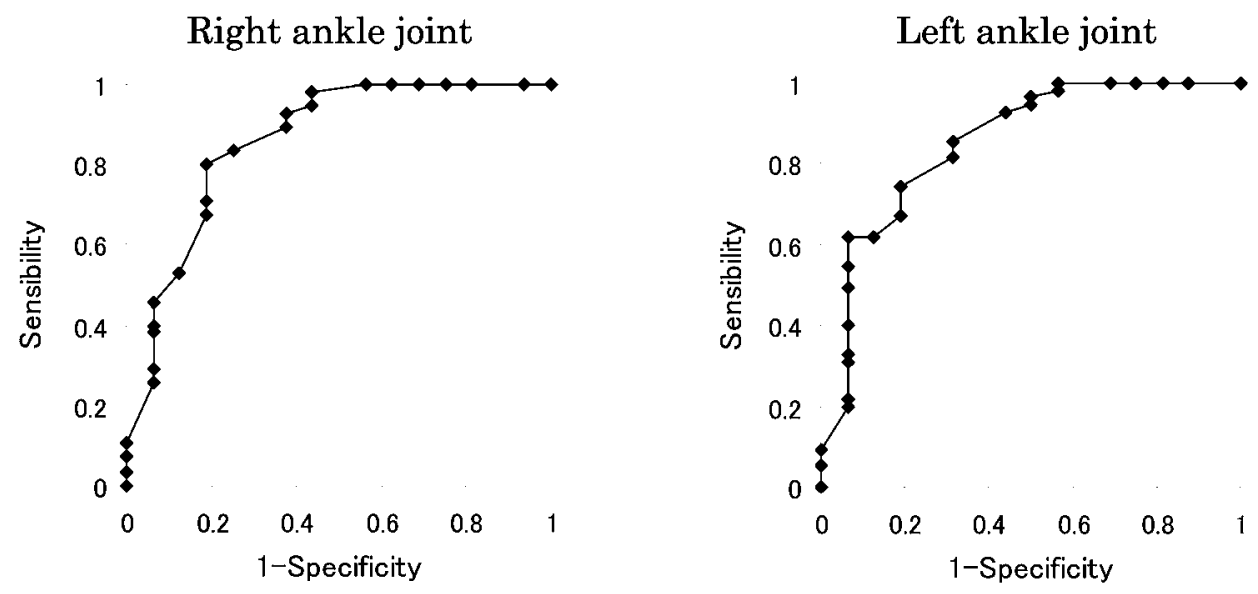

Fig. 1 ROC curve for the possible cutoff points of the flexibilities of the right and left ankle dorsiflexion. The area under the ROC curve was 0.86 for the right side and 0.85 for the left side. The coordinates of the ROC curves show that the ankle dorsiflexion flexibility with weight-bearing lunge test that most accurately represented the deep squatting posture was $10.75 \mathrm{~cm}$ for the right side and $11.25 \mathrm{~cm}$ for the left side.

coordinates of the ROC curves show that the ankle dorsiflexion flexibility with weight-bearing lunge test that most accurately represents the deep squatting posture was $10.75 \mathrm{~cm}$ for the right side (sensitivity, $80 \%$; specificity, $81 \%$ ) and $11.25 \mathrm{~cm}$ for the left side (sensitivity, $80 \%$; specificity, $75 \%$ ).

\section{DISCUSSION}

In this study, $22.5 \%$ of the subjects could not maintain the deep squatting posture. Sugawara et al. ${ }^{10)}$ reported that approximately $20 \%$ of their Japanese subjects, 113 young and 79 middle-aged individuals, could not maintain the deep squatting posture. However, our study was conducted with only young Japanese males. A detailed study on people of both sexes belonging to different races and age groups should be conducted in the future.

Various researchers state that the ability to adopt the deep squatting posture is related to ankle dorsiflexion flexibility ${ }^{10,11)}$. However, since the deep squatting posture has also been shown to be related to anthropometric characteristics and the flexibilities of other joints, it was necessary to determine the factors which influence the deep squatting posture. Therefore, we aimed to determine the intrinsic factors that influence the deep squatting posture. The discriminant analysis conceptually revealed that the deep squatting posture is significantly correlated with ankle dorsiflexion flexibility and body weight.

We think that the increase in ankle plantar flexion moment with the increase in the anteversion angle of the shank is the most influential intrinsic factor for the deep squatting posture. The ankle plantar flexion moment causes front rotation of the shank and helps to stabilize the center of gravity, thus enabling the maintenance of balance.

Adopting the deep squatting posture requires three-dimensional movement of the ankle joints. In this study, we instructed the subjects on the positioning of the foot, and only movement in the sagittal plane was allowed. We think that lowering the degree of freedom of the lower extremities lead to the moderate discrimination percentage obtained by this evaluation method. In order to maintain balance in the case of limitation of ankle dorsiflexion, it is necessary to move the center of gravity forward by compensating with trunk and hip joint flexion. However, in this posture, it is impossible to keep the thigh in contact with the calf. Thus, limitation of ankle dorsiflexion makes it impossible to adopt the deep squatting posture. Body weight is associated with the deep squatting posture only to a slight extent. BMI was found to be correlated to the maximal knee flexion during squatting ${ }^{12)}$. Body weight and BMI are strongly related. We think that increase of body weight also influences the knee flexion, thus making it impossible to keep the thigh in contact with the calf 
and to adopt the deep squatting posture. However, the association could not be elucidated in detail because the thickness of the thigh and calf and percentage of body fat were not measured in this study.

A weight-bearing lunge of less than $11 \mathrm{~cm}$ indicates limitation of ankle dorsiflexion ${ }^{7,8}$. Limitation of ankle dorsiflexion is associated with gait parameters ${ }^{3)}$ and balance function ${ }^{4}$. In addition, it is considered to be a risk factor of falls for older people ${ }^{5)}$ and sports injuries ${ }^{6-8)}$. Therefore, the measurement method described above is expected to enable the prediction of the risk factor, thus leading to the prevention of falls, sports injuries, etc. The method does not require any specific measuring instrument, meaning that the skill level and experience of the evaluator were not significant for the measurements. This study describes an easy and objective measurement of ankle dorsiflexion for physical therapy practice.

\section{REFERENCES}

1) Schenkman M, Berger RA, Riley PO, et al.: Wholebody movements during rising to standing from sitting. Phys Ther, 1990, 70: 638-651.

2) Protopapadaki A, Drechsler WI, Cramp MC, et al.: Hip, knee, ankle kinematics and kinetics during stair ascent and descent in healthy young individuals. Clin Biomech, 2007, 22: 203-210.

3) Crosbie J, Green T, Refshauge K: Effects of reduced ankle dorsiflexion following lateral ligament sprain on temporal and spatial gait parameter. Gait Posture, 1999, 9: 167-172.

4) Gajdosik RL: Relation of age and passive properties of an ankle dorsiflexion stretch to the timed one-leg stance test in older women. Percept Mot Skills, 2006, 103: $177-182$.
5) Menz HB, Morris ME, Lord SR: Foot and ankle risk factors for falls in older people: A prospective study. $\mathrm{J}$ Gerontol A Biol Sci Med Sci, 2006, 61: 866-870.

6) De Noronha M, Refshauge KM, Herbert RD, et al.: Do voluntary strength, proprioception, range of motion, or postural sway predict occurrence of lateral ankle sprain? Br J Sports Med, 2006, 40: 824-828.

7) Gabbe BJ, Finch CF, Wajswelner H, et al.: Predictors of lower extremity injuries at the community level of Australian football. Clin J Sport Med, 2004, 14: 56-63.

8) Gabbe BJ, Bennell KL, Finch CF: Why are older Australian football players at greater risk of hamstring injury? J Sci Med Sport, 2006, 9: 327-333.

9) Chung MK, Lee I, Kee D: Effect of stool height and holding time on postural load of squatting postures. Int J Ind Ergon, 2003, 32: 309-317.

10) Sugawara M, Sugita S, Shimada T: Relationship between squatting posture for excretion and movements of the hip and ankle joints in Japanese women. Structure and Function, 2005, 3: 43-49(in Japanese).

11) Zelle J, Barink M, Loeffen R, et al.:Thigh-calf contact force measurements in deep squatting knee flexion. Clin Biomech, 2007, 22: 821-826.

12) Hemmerich A, Brown H, Smith S, et al:: Hip, knee, and ankle kinematics of high range of motion activities of daily living. J Orthop Res, 2006, 24: 770-781.

13) Torii S: Management and prevention for injuries of adolescent athletes in track and field. Orthop Surg Traumatol, 2000, 43: 1311-1318 (in Japanese).

14) Harvey D: Assessment of the flexibility of elite athletes using the modified Thomas test. Br J Sports Med, 1998, 32: 68-70.

15) Sato H, Maruyama H, Hiiragi $Y$, et al.: Relationship between improvement of FFD by hold-relax on pelvis motion and initial length of hamstrings. Rigakuryoho Kagaku, 2005, 20: 283-287 (in Japanese).

16) Bennell KL, Talbot RC, Wajswelner H: Intra-rater and inter-rater reliability of a weight-bearing lunge measure of ankle dorsiflexion. Aust J Physiother, 1998, 44: 175-180. 